\title{
Dapagliflozin Ameliorates Glycemic State, Lipid Profile and Renal Functions in Type 2 Diabetic Rats
}

\author{
Sohair A. Saleh ${ }^{\text {a }}$, Magda A. Mansour ${ }^{\text {b }}$, Suzan M. Hazzaa ${ }^{\text {a }}$, Amal G. Younis ${ }^{\text {a }}$, Dalia F. El Agamy ${ }^{\text {a }}$
}

a

Department of Physiology,

Faculty of Medicine, Menoufia University, Menoufia, Egypt.

b Department of Histology, Faculty of Medicine, Menoufia University, Menoufia, Egypt.

Correspondence to:

Amal G. Younis, Department of Physiology, Faculty of Medicine, Menoufia University, Menoufia, Egypt

Email:

amalgama1079@gmail.com

Received: 1 June, 2020

Accepted: 25 July, 2020
Abstract

Background: Diabetic kidney disease is the leading cause of chronic kidney disease worldwide. Sodium glucose cotransporter-2 inhibitors may provide a recent solution with better control. Aim: to study the effect of dapagliflozin on glycemic state, lipid profile, renal functions, renal histopathology and some underlying possible mechanisms in type 2 diabetic rats. Methods: Fifty adult male albino rats were divided into five groups: Non-diabetic, diabetic non-treated, diabetic metformintreated, diabetic dapagliflozin-treated and diabetic combined metformin $\&$ dapagliflozin-treated. Type 2 diabetes was induced by high fat dietlow dose streptozotocin. Metformin or dapagliflozin were given. RBF and RVR were measured. Fasting serum glucose, HbA1c, serum insulin, lipid profile, renal function tests, TAC, MDA and TNF- $\alpha$ were measured then HOMA-IR, LDL-C \& creatinine clearance were calculated. Histopathological examination of kidney sections was performed. Results: Hyperglycemia, hyperinsulinemia, insulin resistance, dyslipidemia, impairment of renal functions, altered oxidative and inflammatory modulators status, and changes in renal morphology were observed in diabetic rats. Individual treatment with metformin or dapagliflozin significantly improved those parameters however, better improvement in renal functions was observed in dapagliflozin as compared to metformin-treated groups. Diabetic combined metformin $\&$ dapagliflozin-treated group revealed significant improvement in glycemic state, renal function, TNF- $\alpha$ and oxidative stress as compared to dapagliflozin-treated group. Conclusion: Although metformin is better than dapagliflozin in glycemic control, dapagliflozin is more renoprotective than metformin. Combined dapagliflozin and metformin treatment resulted in significant improvement in glycemic state and renal functions in diabetic rats which is better than individual treatment. dapagliflozin had a complementary effect to metformin treatment in T2DM.

Key words: Dapagliflozin, Renal functions, Sodium glucose cotransporter-2 inhibitor, Type 2 diabetes 


\section{Introduction:}

The incidence of diabetes is increasing worldwide. Diabetic nephropathy (DN) is a common complication of type 1 and type 2 diabetes that has become the leading cause of end-stage renal disease. The pathophysiology of DN comprises an interaction of metabolic factors, such as glucose-dependent pathways, and hemodynamic factors, such as activation of renin-angiotensin system (RAS) and endothelin-I (ET-1), in addition to inflammatory and other alternative pathways $[1,2]$.

Activation of the RAS leads to elevation of angiotensin II levels which subsequently cause efferent arteriolar vasoconstriction, hyperfiltration, increased albuminuria and nephropathy [2]. ET-1 has been implicated in vasoconstriction, renal injury, mesangial proliferation, glomerulosclerosis, fibrosis and inflammation [3].

Hyperglycemia leads to generation of advanced glycation products, reactive oxygen species and inflammatory mediators that activate hyperglycemia-induced injury and induce baleful pathological processes such as mesangial cell proliferation and hypertrophy, excessive accumulation of extracellular matrix proteins and thickening of the glomerular basement membrane that ultimately leads to nodular glomerulosclerosis, a hallmark of DN [4]. Reabsorption of up to $90 \%$ of the filtered glucose is modulated by SGLT2, which is dramatically increased in diabetic subjects and animal models, accounting for the increase in glucose and sodium reabsorption [5, 6]. Increased sodium reabsorption in the proximal tubules initiates hyperfiltration; reduced delivery of sodium to the macula densa leads to reduction of adenosine generation in the juxtaglomerular apparatus leading to enhanced afferent arteriolar vasodilatation, and subsequently increased renal blood flow and glomerular filtration rate (GFR) via the tubuloglomerular feedback (TGF) [7].

Albuminuria and urinary N-acetyl- $\beta$-Dglucosaminidase (NAG) are sensitive indicators for early detection of renal insult in diabetes. Creatinine clearance correlates with GFR and is an indicator for renal functions. Serum cystacin $\mathrm{C}$ is a protein produced by all nucleated cells and is completely catabolized in the proximal renal tubule, cystacin $\mathrm{C}$ has shown promise as a replacement for serum creatinine in estimation of GFR as it is not affected by gender, age, race, protein intake, or muscle mass, unlike serum creatinine [32].

Over the past two decades, treatment of $\mathrm{DN}$ has been primarily centered on controlling 
hyperglycemia and hypertension and inhibiting RAS. SGLT2 inhibitors represent a promising therapeutic approach to prevent and improve nephropathy among patients with type 2 diabetes. The aim of the present investigation was to study the effect of SGLT2 inhibitor (Dapagliflozin) on glycemic state, lipid profile, renal functions, renal histopathology and some underlying possible mechanisms in type 2 diabetic rats.

\section{Materials and Methods:}

Animals and experimental design

It is a prospective study conducted during the period from 20/5/2019 to 20/7/2019 in the laboratories of the faculty of medicine at Menoufia University on fifty adult male albino rats of local strain (150-200 grams each). Rats were housed (5 per cage) in fully ventilated cages $(80 \times 40 \times 30 \mathrm{~cm})$, at room temperature, with natural day/night cycle and free access to water. They were fed with rat normal pellet diet and tap water for 1 week for acclimatization before experiments. The protocol of this study has been approved by Research Ethics Committee of Faculty of Medicine, Menoufia University, Egypt.

After acclimatization rats were divided equally into the following five experimental groups $(\mathrm{n}=10)$ : group I, non-diabetic group (ND) which included normal rats with fasting blood glucose less than $110 \mathrm{mg} / \mathrm{dL}$ [8], group II, diabetic non-treated group (D) where type 2 diabetes was induced by high fat diet (HFD; $58 \%$ fat, $25 \%$ protein and $17 \%$ carbohydrate, as a percentage of total $\mathrm{kcal}$ ) as described by Srinivasan et al. (Table 1) for 4 weeks [9]. Single intraperitoneal injection of streptozotocin (STZ) at a dose of $35 \mathrm{mg} / \mathrm{kg}$, dissolved in distilled water, was administered $[10,11]$.

Diabetes mellitus was confirmed 3 days after STZ injection by measuring tail vein fasting blood glucose levels using glucometer (ACCU-CHEK) in overnight fasted rats. Only rats with fasting blood glucose level $\geq 160$ $\mathrm{mg} / \mathrm{dl}$ were considered diabetic [12]. The diabetic rats were allowed to continue to feed on HFD until the end of the experiment.

Group III, diabetic metformin-treated group (D+MET), where induction of type 2 diabetes was done as group II, then diabetic rats were treated with metformin (250 $\mathrm{mg} / \mathrm{kg} /$ day $)$ dissolved in distilled water given 6 days per week by oral gavage for 4 weeks [10]. Group IV, diabetic dapagliflozin-treated group (D+DAPA), where induction of type 2 diabetes was done, then they were treated with dapagliflozin (1 $\mathrm{mg} / \mathrm{kg} /$ day $)$ dissolved in distilled water given 6 days per week by oral gavage for 4 weeks [13]. Group V, diabetic 
combined metformin \& dapagliflozin-treated group (D+MET+DAPA), where induction of type 2 diabetes was done then, they were treated with both metformin $(250 \mathrm{mg} / \mathrm{kg} /$ day $)$ \& dapagliflozin (1 $\mathrm{mg} / \mathrm{kg} /$ day $)$ dissolved in distilled water given 6 days per week by oral gavage for 4 weeks.

At the end of the experimental period, 24-h urine sample was collected for each rat alone by using a metabolic cage to measure urine volume $(\mathrm{ml} / \mathrm{min})$. After that, fasted rats were anesthetized and renal artery was exposed to measure renal blood flow (RBF) velocity and renal vascular resistance (RVR). Then, fasting blood samples were collected directly from the abdominal aorta [11]. Urine and blood samples were used for estimation of: fasting serum glucose, HbA1c, serum insulin, TC, TG, HDL-C, renal function tests (BUN, serum creatinine, serum cystatin $\mathrm{C}$, urinary creatinine, urinary albumin \& urinary NAG) then homeostasis model assessment for insulin resistance (HOMA-IR), LDL-C \& creatinine clearance ( $\mathrm{ml} / \mathrm{min})$ were calculated. Also, TAC, MDA and TNF- $\alpha$ were measured. Finally, both kidneys were fixed in $10 \%$ formalin for histopathological examination.

\section{Urine and blood sampling}

Each rat was placed in an individual metabolic cage for urine collection which was placed over a funnel; the top of the funnel was covered by wire mesh to avoid fecal contamination of urine. During urine collection the animal received free access to water but no food was given to avoid contamination [14]. The total voided urine during the next 24-h was measured to calculate urine volume $(\mathrm{ml} / \mathrm{min})$. Urine samples were centrifuged at 3000 rotation per minute for 15 minutes to remove any particulates and stored at $-20^{\circ} \mathrm{C}$ until use for biochemical analysis.

Fasting blood samples were collected directly from the abdominal aorta using sterilized plastic fine cannula 20 [11] in 2 clean graduated tubes; the first EDTA tube for estimation of HbA1c and the second was left for clotting at room temperature, then centrifuged at 3000 rotation per minute for 15 minutes and the supernatant serum was collected in dry tubes and was frozen at $-80^{\circ} \mathrm{C}$ until use for biochemical analysis.

\section{Biochemical analysis}

Determination of fasting serum glucose, HbA1c, lipid profile, BUN, TAC, MDA and serum \& urinary creatinine:

Fasting serum glucose (mg/dl), HbAlc (\% of normal), TC (mg/dl), TG (mg/dl) and HDL-C $(\mathrm{mg} / \mathrm{dl}), \mathrm{BUN}(\mathrm{mg} / \mathrm{dl})$, TAC $(\mu \mathrm{mol} / \mathrm{L}), \mathrm{MDA}$ $(\mu \mathrm{mol} / \mathrm{L})$ and serum \& urinary creatinine 
$(\mathrm{mg} / \mathrm{dl})$ levels were determined by enzymatic colorimetric method using test reagent kits (Bio-diagnostic Company, Egypt) according to the manufacturer's instructions $[8,15]$. Then LDL-C was calculated using the following formula: $\mathrm{LDL}-\mathrm{C}=\mathrm{TC}-(\mathrm{HDL}-\mathrm{C}+$ TG / 5) [15]. Also, Creatinine clearance was calculated using the following formula: creatinine clearance $(\mathrm{ml} / \mathrm{min})=$ [Urinary creatinine $(\mathrm{mg} / \mathrm{dl}) \times$ Urine flow rate $(\mathrm{ml} / \mathrm{min})] /$ Serum creatinine $(\mathrm{mg} / \mathrm{dl})$ [13].

Determination of serum insulin, TNF- $\alpha$, and cystatin $c$ and urinary NAG and albumin:

Insulin $(\mu \mathrm{U} / \mathrm{ml})[16]$, TNF- $\alpha(\mathrm{pg} / \mathrm{ml})$, serum cystatin c (mg/l), urinary NAG (IU/l) (Sigma Company, USA) and urinary albumin (mg/24h; Bio-diagnostic Company, Egypt) levels were determined using enzyme-linked immunosorbent assay kits according to the manufacturer's instructions. Insulin resistance was calculated by HOMA-IR using the following formula: HOMA-IR = [fasting glucose $(\mathrm{mg} / \mathrm{dl}) \times$ fasting insulin $(\mu \mathrm{U} / \mathrm{ml})] /$ 405) [17].

\section{Measurement of RBF velocity and RVR}

Each rat was anesthetized with intraperitoneal injection of thiopental sodium at a dose of 50 $\mathrm{mg} / \mathrm{kg}$ by, this dose was usually satisfactory.
A booster dose of about $50-100 \mathrm{mg} / \mathrm{kg}$ was needed in few cases, then the animal was laid on its back and anterior abdominal wall was opened through midline laparotomy, after cleaning the skin and shaving the hair, to expose the renal artery. After setting the mode of pulsed blood flowmeter (Doppler, HADECO, Japan) we used ultrasonic probe pressed softly to the measured area at an angle of $40^{\circ}-50^{\circ}$.

After hearing optimal sound, we waited for 5 seconds without moving the probe then pressed the freeze key to freeze the wave form and RBF velocity and RVR were measured in all groups.

\section{Histopathological samples preparation}

Serial $4-\mu \mathrm{m}$ thick sections were collected from paraffin embedded kidneys followed by $\mathrm{H} \& \mathrm{E}$ and Mallory trichrome staining to assess histological changes. Slides were examined under a light microscope at a magnification of X400 and analyzed by a pathologist blinded to the groups

\section{Statistical analysis}

Data were expressed as mean \pm SEM. Data were analyzed using a personal computer with SPSS, version 20 (SPSS Inc., Chicago, Illinois, USA). Statistical significance was taken as $\mathrm{P}<0.05$ for all experiments, using one-way analysis of variance (ANOVA) 
followed by Tukey's multiple comparison test to judge the difference between various groups for the parametric parameters.

\section{Results}

Fasting serum glucose, HbA1c, fasting serum insulin and HOMA-IR in D group were significantly higher $(P<0.05)$ when compared to their corresponding levels in ND group, while these parameters in D+MET, D+DAPA and D+MET+DAPA groups were significantly lower $(P<0.05)$ when compared to their corresponding levels in D group. The same parameters were significantly higher in D+DAPA group when compared to their corresponding levels in ND $(\mathrm{P}<0.05)$ and $\mathrm{D}+\mathrm{MET}$ groups $(\mathrm{P}<0.05)$.

The same parameters were significantly lower in $\mathrm{D}+\mathrm{MET}+\mathrm{DAPA}$ group when compared to their corresponding levels in D+MET $(\mathrm{P}<0.05)$ and $\mathrm{D}+\mathrm{DAPA}$ groups $(\mathrm{P}<0.05)$ (Table 2).

Serum TC, Serum TG and LDL-C in D group were significantly higher $(P<0.05)$ when compared to their corresponding levels in ND group, while these parameters in D+MET and D+MET+DAPA groups were significantly lower $(P<0.05)$ when compared to their corresponding levels in D group. In D+DAPA group serum TC and LDL-C were insignificantly changed $(P>0.05) \&$ serum TG level was significantly lower $(P<0.05)$ when compared to their corresponding level in $\mathrm{D}$ group. These parameters were significantly higher $(P<0.05)$ in D+DAPA group when compared to their corresponding levels in ND and D+MET groups. These parameters were significantly lower $\quad(P<0.05) \quad$ in D+MET+DAPA group when compared to their corresponding levels in D+MET and D+DAPA groups (Table 3).

Serum HDL-C in D group was significantly lower $(P<0.05)$ when compared to the corresponding level in ND group, while this parameter in D+MET, D+DAPA and D+MET+DAPA groups was significantly higher $(\mathrm{P}<0.05)$ when compared to the corresponding level in D group. HDL-C was significantly lower in D+DAPA group when compared to the corresponding level in D+MET and D+MET+DAPA groups $(\mathrm{P}<0.05)$, while this parameter was significantly higher $(\mathrm{P}<0.05) \quad$ in D+MET+DAPA group when compared to their corresponding levels in D+MET and D+DAPA groups (Table 3).

Serum creatinine, BUN, serum cystatin C, urinary NAG, urinary albumin, and RVR in D group were significantly higher $(\mathrm{P}<0.05)$ when compared to their corresponding levels in ND group. These parameters except urinary 
albumin were significantly lower $(\mathrm{P}<0.05)$ in D+MET group when compared to their corresponding levels in D group. In D+DAPA group these parameters were significantly higher $(P<0.05)$ when compared to their corresponding levels in ND group and significantly lower $(P<0.05)$ when compared to their corresponding levels in $\mathrm{D}$ and D+MET groups except urinary albumin that was still insignificantly changed when compared to $\mathrm{D}+\mathrm{MET}$ group $(\mathrm{P}>0.05)$. In $\mathrm{D}+\mathrm{MET}+\mathrm{DAPA}$ group these parameters were significantly lower $(P<0.05)$ when compared to their corresponding levels in D, D+MET and D+DAPA groups.

Creatinine clearance and RBF velocity in D group were significantly lower $(\mathrm{P}<0.05)$ when compared to their corresponding levels in ND group. These parameters in D+MET, $\mathrm{D}+\mathrm{DAPA}$ and $\mathrm{D}+\mathrm{MET}+\mathrm{DAPA}$ groups were significantly higher $(\mathrm{P}<0.05)$ when compared to their corresponding levels in D group. However, D+DAPA group showed significantly higher level of these parameters when compared to the corresponding levels in $\mathrm{D}+\mathrm{MET}$ group $(\mathrm{P}<0.05)$. In $\mathrm{D}+\mathrm{MET}+\mathrm{DAPA}$ group these parameters were significantly higher $(P<0.05)$ when compared to their corresponding levels in D, D+MET and D+DAPA groups (Table 4).
Serum TAC in D group was significantly lower $(\mathrm{P}<0.01)$ when compared to the corresponding level in ND group. Serum TAC in D+MET, D+DAPA and D+MET+DAPA groups was significantly higher $(\mathrm{P}<0.05)$ when compared to the corresponding level in D group. Serum TAC in D+DAPA group was significantly higher $(\mathrm{P}<0.05)$ when compared to the corresponding level in D+MET group. Serum TAC in D+MET+DAPA group was significantly higher $(\mathrm{P}<0.05)$ when compared to the corresponding level in D+MET and D+DAPA groups (Table 5).

Serum MDA and TNF- $\alpha$ in D group were significantly higher $(\mathrm{P}<0.01)$ when compared to their corresponding levels in ND group, while these parameters in D+MET, D+DAPA and D+MET+DAPA groups were significantly lower $(\mathrm{P}<0.01)$ when compared to their corresponding levels in D group. Serum MDA was insignificantly lower $(\mathrm{P}>0.05)$, while TNF- $\alpha$ was significantly lower $(\mathrm{P}<0.01)$ in D+DAPA and D+MET+DAPA groups when compared to the corresponding level in D+MET group. Serum MDA was insignificantly lower ( $\mathrm{P}>0.05)$, while TNF- $\alpha$ was significantly lower $(\mathrm{P}<0.01)$ in $\mathrm{D}+\mathrm{MET}+\mathrm{DAPA}$ group when compared to the corresponding level in D+DAPA group (Table 5).

Histopathological examination of kidney 
tissue in diabetic group revealed marked histological changes in the form of interstitial fibrosis. D+MET group showed moderate to good improvement of histological pictures; most of renal tubules appeared quite normal, while others showed degeneration of their cell lining and cystic dilation of their lumen, most of renal glomeruli appeared normal, while others appeared with clumped glomerular capillary tuft with increased mesangial matrix and hyperplasia of mesangial cells and minimal amount of connective tissue fibers in the renal interstitium. D+DAPA group showed good glomerulosclerosis, tubular cell necrosis, hyalinosis, mononuclear infiltration, and improvement of histological pictures; the renal tissues showed nearly normal renal glomeruli and renal tubules and minimal amount of connective tissue fibers in the renal interstitium. D+MET+DAPA group showed marked improvement of histological pictures. The renal tissues showed nearly normal renal glomeruli and normal renal tubules and very minimal amount of connective tissue fibers in the renal interstitium like the ND group (fig. $1)$.

Table (1): Constituents of the HFD

Ingredients

Powdered normal pellet diet (Egyptian market)

Lard (Egyptian market)

Casein (Difco, Becton Dickinson, France)

Cholesterol (Sigma-Aldrich, Cairo, Egypt)

Vitamin and mineral mix (Sigma-Aldrich, Cairo, Egypt)

dl-Methionine (Sigma-Aldrich, Cairo, Egypt)

Yeast powder (Egyptian market)

Sodium chloride (Egyptian market)
Diet (g/kg) 
Table (2):- Fasting blood glucose, HbA1c, fasting serum insulin and HOMA-IR in all studied groups.

\begin{tabular}{lccccc}
\hline & ND & D & D+MET & D+DAPA & D+MET+DAPA \\
\hline Fasting serum glucose $(\mathrm{mg} / \mathrm{dl})$ & $90.5 \pm 0.75$ & $381 \pm 1.28^{*}$ & $181.7 \pm 0.9^{* \#}$ & $209.6 \pm 0.92^{* \# \Omega}$ & $102 \pm 0.5^{\# \Omega ¥}$ \\
HbA1c $(\%$ of N.Hb) & $3.49 \pm 0.05$ & $8.45 \pm 0.05^{*}$ & $7.3 \pm 0.04^{* \#}$ & $7.7 \pm 0.04^{* \# \Omega}$ & $5.6 \pm 0.05^{* \# \Omega \neq}$ \\
Fasting serum insulin $(\mu \mathrm{U} / \mathrm{ml})$ & $3 \pm 0.03$ & $10.5 \pm 0.03^{*}$ & $4.2 \pm 0.03^{* \#}$ & $4.4 \pm 0.04^{* \# \Omega}$ & $3.8 \pm 0.03^{* \# \Omega \neq}$ \\
HOMA-IR & $0.67 \pm 0.01$ & $9.89 \pm 0.03^{*}$ & $1.9 \pm 0.01^{* \#}$ & $2.32 \pm 0.02^{* \# \Omega}$ & $1.2 \pm 0.01^{* \# \Omega \neq}$ \\
\hline
\end{tabular}

Data were expressed as mean \pm S.E.M $(\mathrm{n}=10)$. ${ }^{*}$ Significant vs ND group. \# Significant vs D group. $\Omega$ Significant vs $\mathrm{D}+\mathrm{MET}$ group. ¥ Significant vs D+DAPA group. ND: non diabetic group, D: diabetic non treated group, D+MET: diabetic metformintreated group, D+DAPA: diabetic dapagliflozin-treated group, D+MET+DAPA: diabetic combined metformin \& dapagliflozintreated group . HbA1c: Hemoglobin A1c ; HOMA-IR: Homeostatic Model Assessment of Insulin Resistance

Table (3):- Lipid profile (serum TC, serum TG, HDL-C and LDL-C) in all studied groups.

\begin{tabular}{|c|c|c|c|c|c|}
\hline & $N D$ & $D$ & $D+M E T$ & $D+D A P A$ & $D+M E T+D A P A$ \\
\hline Serum TC (mg/dl) & $106 \pm 0.48$ & $261 \pm 0.67^{*}$ & $114 \pm 0.63^{* \#}$ & $262 \pm 0.58^{* \Omega}$ & $118 \pm 0.5^{*} \# \Omega ¥$ \\
\hline Serum TG $(\mathrm{mg} / \mathrm{dl})$ & $64.3 \pm 0.45$ & $163.2 \pm 0.63^{*}$ & $101.7 \pm 0.68^{* \#}$ & $154.2 \pm 0.77^{* \# \Omega}$ & $80.3 \pm 0.31 * \# \Omega ¥$ \\
\hline HDL-C (mg/dl) & $39.7 \pm 0.4$ & $16.9 \pm 0.49^{*}$ & $23 \pm 0.43^{* \#}$ & $19.5 \pm 0.38^{* \# \Omega}$ & $31.7 \pm 0.3 * \# \Omega ¥$ \\
\hline LDL-C (mg/dl) & $53.5 \pm 0.77$ & $211.5 \pm 1^{*}$ & $71.5 \pm 0.92^{* \#}$ & $211.6 \pm 0.57^{* \Omega}$ & $65.5 \pm 0.5 * \# \Omega ¥$ \\
\hline
\end{tabular}

Data were expressed as mean \pm S.E.M $(n=10)$ * Significant vs ND group. \# Significant vs D group. $\Omega$ Significant vs D+MET group. ¥ Significant vs D+DAPA group ND: non diabetic group, D: diabetic non treated group, D+MET: diabetic metformintreated group, D+DAPA: diabetic dapagliflozin-treated group, D+MET+DAPA: diabetic combined metformin \& dapagliflozintreated group. HDL-C: high density lipoprotein-cholesterol; LDL-C: low density lipoprotein-cholesterol.

Table (4):- Renal function parameters (BUN, serum creatinine, creatinine clearance, serum cystatin C, urinary NAG, urinary albumin, RBF velocity and RVR) in all studied groups.

\begin{tabular}{|c|c|c|c|c|c|}
\hline & $N D$ & $D$ & $D+M E T$ & $D+D A P A$ & $D+M E T+D A P A$ \\
\hline BUN (mg/dl) & $24.9 \pm 0.62$ & $65.8 \pm 0.78^{*}$ & $51.5 \pm 0.91^{* \#}$ & $44.5 \pm 0.93^{* \# \Omega}$ & $32.9 \pm 0.5^{* \# \Omega ¥}$ \\
\hline Serum creatinine $(\mathrm{mg} / \mathrm{dl})$ & $0.32 \pm 0.027$ & $1.29 \pm 0.034^{*}$ & $0.76 \pm 0.019^{* \#}$ & $0.6 \pm 0.022^{* \# \Omega}$ & $0.41 \pm 0.01^{* \# \Omega \neq}$ \\
\hline Creatinine clearance $(\mathrm{ml} / \mathrm{min})$ & $6.8 \pm 0.3$ & $1.84 \pm 0.04^{*}$ & $3.13 \pm 0.06^{* \#}$ & $5.58 \pm 0.1^{* \# \Omega}$ & $6.1 \pm 0.2^{* \# \Omega ¥}$ \\
\hline Serum cystatin C (mg/l) & $0.48 \pm 0.016$ & $1.19 \pm 0.024^{*}$ & $0.72 \pm 0.013^{* \#}$ & $0.61 \pm 0.011^{* \# \Omega}$ & $0.55 \pm 0.015^{* \# \Omega \neq}$ \\
\hline Urinary NAG (IU/L) & $46.6 \pm 1$ & $78.4 \pm 1.7^{*}$ & $57.9 \pm 1.3^{* \#}$ & $52.1 \pm 1.2^{* \# \Omega}$ & $47.6 \pm 0.6^{\# \Omega \neq}$ \\
\hline Urinary albumin (mg/24hr) & $10.9 \pm 0.7$ & $20.9 \pm 1.5^{*}$ & $17.5 \pm 0.9^{*}$ & $16.2 \pm 0.8^{* \#}$ & $14.9 \pm 0.5^{* \# \Omega ¥}$ \\
\hline RBF velocity $(\mathrm{cm} / \mathrm{sec})$ & $7.27 \pm 0.08$ & $2.64 \pm 0.05^{*}$ & $4.22 \pm 0.04^{* \#}$ & $5.15 \pm 0.06^{* \# \Omega}$ & $6.6 \pm 0.05^{* \# \Omega \neq}$ \\
\hline RVR (PRU) & $0.73 \pm 0.033$ & $1.09 \pm 0.071^{*}$ & $0.87 \pm 0.07^{* \#}$ & $0.78 \pm 0.07^{* \# \Omega}$ & $0.74 \pm 0.04^{\# \Omega ¥}$ \\
\hline
\end{tabular}

Data were expressed as mean \pm S.E.M $(n=10)$. * Significant vs ND group. \# Significant vs D group. $\Omega$ Significant vs D+MET group. $¥$ Significant vs D+DAPA group. ND: non diabetic group, D: diabetic non treated group, D+MET: diabetic metformin-treated group, D+DAPA: diabetic dapagliflozin-treated group, D+MET+DAPA: diabetic 
combined metformin \& dapagliflozin-treated group. BUN: Blood urea nitrogen, RBF: renal blood flow, RVR: renal vascular resistance.

Table (5):- Serum TAC, serum MDA and serum TNF- $\alpha$ in all studied groups.

\begin{tabular}{lccccl}
\hline & $\boldsymbol{N D}$ & $\boldsymbol{D}$ & $\boldsymbol{D}+\boldsymbol{M E T}$ & $\boldsymbol{D}+\boldsymbol{D A P A}$ & \multicolumn{1}{c}{$\boldsymbol{D + M E T + \boldsymbol { D A P A }}$} \\
\hline $\mathrm{TAC}(\mu \mathrm{mol} / \mathrm{L})$ & $2.77 \pm 0.04$ & $1.42 \pm 0.02^{*}$ & $2.1 \pm 0.07^{* \#}$ & $2.5 \pm 0.08^{* \# \Omega}$ & $2.69 \pm 0.03^{\# \Omega *}$ \\
$\mathrm{MDA}(\mu \mathrm{mol} / \mathrm{L})$ & $2.3 \pm 0.03$ & $4 \pm 0.19^{*}$ & $2.5 \pm 0.04^{\#}$ & $2.4 \pm 0.06^{\#}$ & $2.36 \pm 0.04^{\#}$ \\
$\mathrm{TNF}-\alpha(\mathrm{Pg} / \mathrm{ml})$ & $14.9 \pm 0.4$ & $115.4 \pm 0.7^{*}$ & $86.4 \pm 0.8^{* \#}$ & $81 \pm 0.5^{* \# \Omega}$ & $30.9 \pm 0.5^{* \# \Omega}$ \\
\hline
\end{tabular}

Data were expressed as mean \pm S.E.M ( $\mathrm{n}=10) .{ }^{*}$ Significant vs ND group. \# Significant vs D group. $\Omega$ Significant vs D+MET group. ¥ Significant vs D+DAPA group. ND: non diabetic group, D: diabetic non treated group, D+MET: diabetic metformin-treated group, D+DAPA: diabetic dapagliflozin-treated group, D+MET+DAPA: diabetic combined metformin \& dapagliflozin-treated group. TAC: total antioxidant capacity, MDA: malondialdehyde, TNF- $\alpha$ : tumor necrosis factor- $\alpha$.

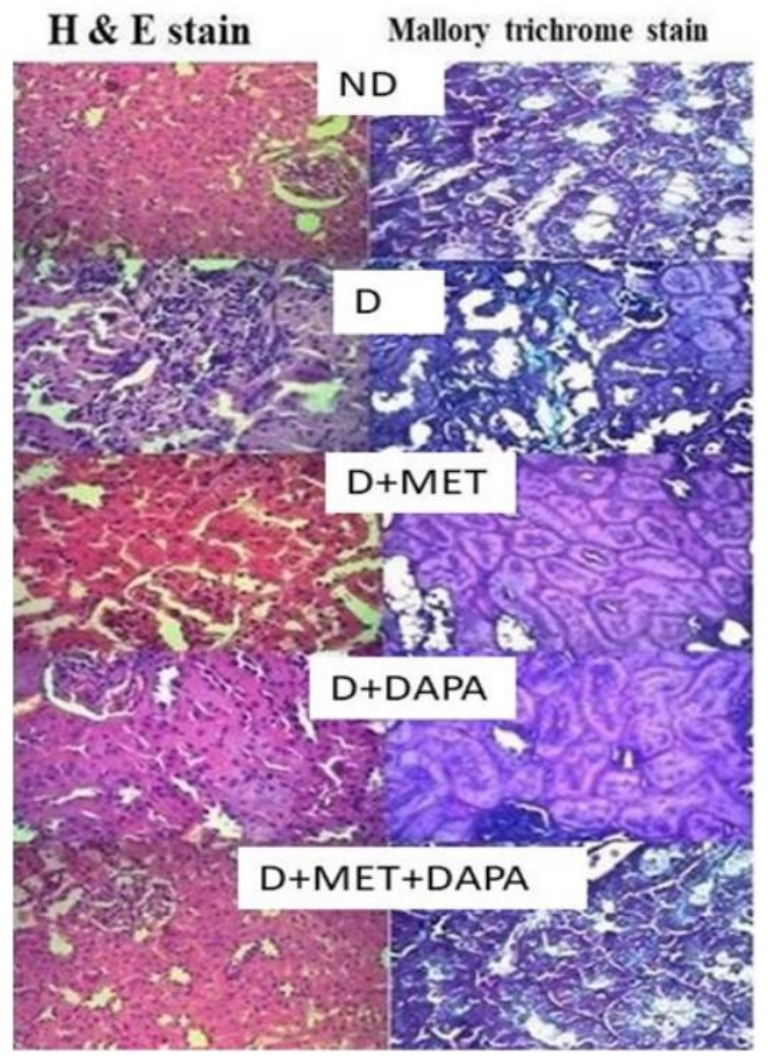

Fig. (1) 


\section{Discussion:}

Diabetes is a major health problem globally approaching an epidemic [18]. Diabetic kidney disease develops in approximately $40 \%$ of diabetic patients and is the leading cause of chronic kidney disease [19]. SGLT2 inhibitors produce glucosuria, reduce plasma glucose concentrations and suppress renal RAS in diabetic rats [20]. We investigated the effects of SGLT2 inhibitor, dapagliflozin, on the glycemic state, lipid profile, and renal functions in type 2 diabetic rats and recorded the possible associated changes in serum MDA, TAC and TNF- $\alpha$.

The establishment of T2DM model in rats was achieved by feeding HFD followed by low dose of STZ injection (HFD/STZ) [21, 22]. In our study, D rats developed hyperglycemia, hyperinsulinemia, dyslipidemia, and insulin resistance. Srinivasan et al. reported that HFD/STZ is a cheap and easy method that can effectively induce a rat model that mimic T2DM in human. Rats treated with HFD/STZ developed various components of T2DM such as insulin resistance, hyperglycemia, hyperinsulinemia, dyslipidemia, and hypertension [22, 23].

We observed a significant decrease in fasting blood glucose, HbA1c, serum insulin and insulin resistance in D+MET group as compared to D group. There was also a significant decrease in serum TG, TC and LDL-C and significant increase in HDL-C in the same group. Similar results were observed in a previous study [24].

We also studied the effects of a new class of oral anti-hyperglycemic medications that is US Food and Drug Administration approved in type 2 diabetes, dapagliflozin [25]. Dapagliflozin is a SGLT2 inhibitor that improved the hyperglycemic, insulin resistant, dyslipidemic effects of HFD/STZ type 2 diabetes. By blocking SGLT2 in the early proximal tubules of the kidney, SGLT2 inhibitors lowers renal glucose reabsorption and blood glucose levels via a predominantly insulin-independent mechanism, thereby improving blood glucose control, lowering $\mathrm{HbA1c}$ and enhancing liver insulin sensitivity without inducing hypoglycemia in diabetic patients $[26,27]$. However, these effects were less significant than those observed with metformin monotherapy. Improvement in glucose control decreased TG and increased HDL-C levels and decreased insulin resistance [28].

In the present investigation, HFD/STZ 
diabetic rats showed increased serum MDA, TAC, and TNF- $\alpha$. Oxidative stress driven by hyperglycemia and dyslipidemia induces inflammatory response; hyperglycemia was shown to increase the circulating TNF- $\alpha$ and impairs insulin signaling which are important factors in the development of microvascular diabetic complications including nephropathy. Excess ROS generation leads to cell damage $[29,30]$.

In the present investigation, metformin and dapagliflozin monotherapy significantly reduced serum MDA and TNF- $\alpha$ and significantly enhanced serum TAC levels. Metformin was reported to exhibit antioxidant and anti-inflammatory activities by suppressing mitochondrial respiration that increases the antioxidant enzyme activities and diminishes ROS production in diabetic rats. It also suppresses AGEs production [24]. Furthermore, dapagliflozin attenuated early diabetic renal injury by improvement of antioxidant mechanisms and modulating inflammatory processes in diabetic rats [31].

In the present study, we observed significant alterations in renal functions in D group comparing to ND group; there was significant elevation in urinary NAG and albumin, and serum BUN, creatinine, and cystatin C. In addition, creatinine clearance was significantly decreased indicating renal damage.

The pathogenesis of DN has been tied to a number of pathways which are directly or indirectly linked to hyperglycemia, hyperlipidemia, inflammation and oxidative stress $[33,34]$.

Consistent with previous studies [35], the D+MET group showed significant improvement in renal function tests that can be explained by amelioration of the glycemic and lipidemic states, and anti-inflammatory and antioxidant effects of metformin. Dapagliflozin treatment had also improved renal functions in HFD/STZ diabetic rats through inhibition of inflammation, fibrosis, endoplasmic reticulum stress and apoptosis, and by reducing the accumulation of lipids in the kidneys of HFD-fed rats [36]. In addition, consistent with our results, dapagliflozin had greater efficacy than metformin for attenuating renal dysfunction at least in part by reducing renal oxidative stress and modulating renal insulin signaling pathways [13].

Induction of glomerular hyperfiltration in diabetes is crucial for the progression of renal injury. In addition to RAS activation, hyperglycemia results in increased proximal 
tubular $\mathrm{Na}^{+}$reabsorption mediated by SGLT2, this decreases $\mathrm{Na}^{+}$delivery to the early distal tubule with consequent reduction in ATP and adenosine production resulting in TGFdependent vasodilation of the afferent arteriole and thus hyperfiltration and increased RBF [37-39]. Initial stages of DN are characterized by altered renal hemodynamics with increased intraglomerular pressure and glomerular hyperfiltration mainly due to reduced TGF and dilation of afferent arterioles [7]. This can lead to glomerular damage, release of cytokines and growth factors, resulting in tissue proliferation and fibrosis [40].

We observed significant decrease in RBF velocity and increased RVR, and altered renal histological structure in D group. Decreased RBF velocity indicates a decrease in renal blood flow as changes in velocity are directly and reliably proportional to changes in the volume flow [41]. Our results are in accordance with Freitas et al. who reported that STZ-diabetic rats showed significant reduction in renal blood flow and significant elevation of renal vascular resistance [42]. Reduced insulin-stimulated NO production was observed in insulin-resistant states. Hyperglycemia increases intrarenal ANG II, a key mediator of the increased oxidative stress that increases oxidation of NO and decreases NO-dependent vasodilatation resulting in endothelial dysfunction and altered smooth muscle of renal vessels leading to increased RVR and ultimately reduced $\operatorname{RBF}[39,43]$.

In agreement with previous studies [44, 45], metformin-treated diabetic rats showed significant improvement in renal hemodynamics and increased RBF explained by the hypoglycemic, antioxidant, and antiinflammatory properties. Dapagliflozin-treated diabetic rats have also shown significant increase in RBF velocity and significant decrease in RVR. SGLT2 inhibitors are considered as a very promising method for prevention of renal hyperfiltration and progression of $\mathrm{DN}$ via control of hyperglycemia and partial suppression of renal RAS and oxidative stress [46, 20]. Increased SGLT2 gene expression and glucose-transport capacity was reported in patients with type 2 diabetes [47].

SGLT2 inhibition should result in an increased delivery of sodium to the macula densa, a consequent increase in adenosine release, resulting in vasoconstriction of the afferent arteriole leading to a reduction in RBF and GFR [38]. Additionally, dapagliflozin was more effective than metformin in improving $\mathrm{RBF}$ and RVR as observed in the present study.

The decline in renal function was associated 
with altered renal histological structure including, glomerulosclerosis, hyalinosis, and interstitial fibrosis in D group. Jain and Saha have observed mesangial hypercellularity and capillary basement membrane thickening in the kidney of diabetic rats that was explained by increased oxidative stress, stimulation of RAS and expression of growth factors and inflammatory cytokines [48]. Morphological changes as mesangial expansion and thickening of the glomerular and tubular basement membrane, as well as the typical glomerulosclerosis with mesangial nodular lesions can also be attributed to the impact of hyperglycemia and hyperfiltration [49]. Glomerular hyperfiltration, by applying mechanical stress, leads to podocyte loss, focal segmental glomerulosclerosis, and increased delivery and reabsorption of small and large molecular weight solutes with ensuing hypertrophy, tubulointerstitial inflammation and fibrosis [50].

Metformin partially improved renal histological changes in the D+MET group. Our findings were in agreement with Zhang et al., who observed that metformin attenuated the morphological changes associated with $\mathrm{DN}$ in rats, due to glycemic control, lipid metabolism, and antioxidant and antiinflammatory functions [35]. Nevertheless, dapagliflozin significantly restored and normalized renal histological structure in the D+DAPA group. The renoprotective effects of dapagliflozin are likely direct effects through normalization of glomerular hemodynamics and restoration of TGF in addition to glycemic control, antioxidant and anti-inflammatory effects [51].

In agreement with previous studies [13, 31], combined treatment of diabetic rats by both metformin \& dapagliflozin resulted in significant improvement in glycemic state, oxidative stress, inflammatory state, renal functions and renal hemodynamics when compared to D group, D+MET or D+DAPA groups. The improvement in all previously investigated parameters can be explained on the basis of combination of hypoglycemic, antioxidant and anti-inflammatory effects of both drugs.

\section{Conclusion}

The current data support that dapagliflozin is more renoprotective than metformin in type 2 diabetes. The renoprotective properties of dapagliflozin are most probably due to improving glycemic control, antioxidant, antiinflammatory, and hemodynamic effects.

Combined dapagliflozin treatment with metformin resulted in significant improvement of the glycemic state and renal functions in diabetic rats. This improvement was better 
than that of using each of them alone. This may indicate that dapagliflozin had a complementary effect to metformin treatment in T2DM. So, dapagliflozin is a promising adjuvant therapy for T2DM.

\section{References}

1. Cao Z, Cooper ME. Pathogenesis of diabetic nephropathy. J. Diabetes Investig. 2011 2:243-7.

2. Toth-Manikowski S, Atta MG. Diabetic Kidney Disease: Pathophysiology and Therapeutic Targets. J. Diabetes Res. 2015;2015:697010-697010.

3. Anguiano L, Riera M, Pascual J, Soler MJ. Endothelin Blockade in Diabetic Kidney Disease. J. Clin. Med. 2015;4:1171-1192.

4. Kanwar YS, Sun L, Xie P, Liu F-Y, Chen S. A glimpse of various pathogenetic mechanisms of diabetic nephropathy. Annu Rev Pathol. 2011;6:395423.

5. Hinden L, Udi S, Drori A, Gammal A, Nemirovski A, Hadar R et al. Modulation of Renal GLUT2 by the Cannabinoid-1 Receptor: Implications for the Treatment of Diabetic Nephropathy. J. Am Soc Nephrol. 2017;29:434-448.

6. Alicic RZ, Neumiller JJ, Johnson EJ, Dieter B, Tuttle KR. Sodium-Glucose Cotransporter 2 Inhibition and Diabetic Kidney Disease. Diabetes. 2019;68:248257.

7. Sasson AN, Cherney DZ. Renal hyperfiltration related to diabetes mellitus and obesity in human disease. World J Diabetes. 2012;3:1-6.
8. Nayak SS, Pattabiraman TN. A new colorimetric method for the estimation of glycosylated hemoglobin. Clin. Chim. Acta. 1981;109:267-274.

9. Srinivasan K, Patole PS, Kaul CL, Ramarao P. Reversal of glucose intolerance by pioglitazone in high fat diet-fed rats. Method Find Exp Clin. 2004;26:327-333.

10. Subhasree N, Kamella A, Kaliappan I, Agrawal A, Dubey GP. Antidiabetic and antihyperlipidemic activities of a novel polyherbal formulation in high fat diet/streptozotocin induced diabetic rat model. Indian J. Pharmacol. 2015;47:509-513.

11.Al-Hariri M, Eldin TG, Abu-Hozaifa B, Elnour A. Glycemic control and anti-osteopathic effect of propolis in diabetic rats. Diabetes Metab Syndr Obes. 2011;4:377-384.

12.Gandhi S, Srinivasan B, Akarte AS. Aliskiren improves insulin resistance and ameliorates diabetic renal vascular complications in STZ-induced diabetic rats. J Renin-Angio-Aldo S. 2013 14:3-13.

13. Jaikumkao K, Pongchaidecha A, Chueakula N, Thongnak L, Wanchai $\mathrm{K}$, Chatsudthipong $\mathrm{V}$ et al. Renal outcomes with sodium glucose cotransporter 2 (SGLT2) inhibitor, dapagliflozin, in obese insulinresistant model. BBA-Mol Basis Dis. 2018;1864:2021-2033.

14.Daneshgari F, Huang X, Liu G, Bena J, Saffore L, Powell CT. Temporal differences in bladder dysfunction caused by diabetes, diuresis, and treated diabetes in mice. Am J Physiol-Reg I. 2006 290:R1728-R1735.

15. Trinder P. Determination of blood glucose using 4amino phenazone as oxygen acceptor. J Clin Pathol. 1969;22:246-246. 
16.Judzewitsch RG, Pfeifer MA, Best JD, Beard JC, Halter JB, Porte D, Jr. Chronic chlorpropamide therapy of noninsulin-dependent diabetes augments basal and stimulated insulin secretion by increasing islet sensitivity to glucose. J. Clin. Endocrinol. Metab. 1982 55:321-328.

17. Matthews DR, Hosker JP, Rudenski AS, Naylor BA, Treacher DF, Turner RC. Homeostasis model assessment: insulin resistance and beta-cell function from fasting plasma glucose and insulin concentrations in man. Diabetologia 1985;28:412-419.

18. Tabish SA. Is Diabetes Becoming the Biggest Epidemic of the Twenty-first Century? Int. J. Health Sci. 2007;1:V-VIII.

19. Alicic RZ, Rooney MT, Tuttle KR. Diabetic Kidney Disease: Challenges, Progress, and Possibilities. Clin J Am Soc Nephro. 2017;12:20322045.

20.Shin SJ, Chung S, Kim SJ, Lee E-M, Yoo Y-H, Kim J-W et al. Effect of Sodium-Glucose CoTransporter 2 Inhibitor, Dapagliflozin, on Renal Renin-Angiotensin System in an Animal Model of Type 2 Diabetes. PloS one 2016;11:e0165703e0165703.

21.Guo X-X, Wang Y, Wang K, Ji B-P, Zhou F. Stability of a type 2 diabetes rat model induced by high-fat diet feeding with low-dose streptozotocin injection. J. Zhejiang Univ. Sci. B. 2018;19:559-569.

22. Srinivasan K, Viswanad B, Asrat L, Kaul CL, Ramarao P. Combination of high-fat diet-fed and lowdose streptozotocin-treated rat: a model for type 2 diabetes and pharmacological screening. Pharmacol. Res. 2005;52:313-3120.
23.Suman RK, Ray Mohanty I, Borde MK, Maheshwari U, Deshmukh YA. Development of an Experimental Model of Diabetes Co-Existing with Metabolic Syndrome in Rats. Adv. pharmacol. sci. 2016;2016:9463476-9463476.

24. Abdulmalek SA, Balbaa M. Synergistic effect of nano-selenium and metformin on type 2 diabetic rat model: Diabetic complications alleviation through insulin sensitivity, oxidative mediators and inflammatory markers. PloS one 2019;14:e0220779e0220779.

25.Hsia DS, Grove O, Cefalu WT. An update on sodium-glucose co-transporter-2 inhibitors for the treatment of diabetes mellitus. Curr Opin Endocrinol Diabetes Obes. 2017;24:73-79.

26. Vallon V, Sharma K. Sodium-glucose transport: role in diabetes mellitus and potential clinical implications. Curr Opin Nephrol Hy. 2010;19:425431.

27. Gallo LA, Wright EM, Vallon V. Probing SGLT2 as a therapeutic target for diabetes: Basic physiology and consequences. Diabetes Vasc Dis Re. 2015;12:7889.

28. Abbate SL, Brunzell JD. Pathophysiology of hyperlipidemia in diabetes mellitus. J Cardiovasc Pharmacol. 1990;16 S1-S7.

29. Bin-Jumah MN. Antidiabetic Effect of Monolluma quadrangula Is Mediated via Modulation of Glucose Metabolizing Enzymes, Antioxidant Defenses, and Adiponectin in Type 2 Diabetic Rats. Oxid Med Cell Longev. 2019;2019:6290143.

30. Navarro JF, Mora-Fernandez C. The role of TNFalpha in diabetic nephropathy: pathogenic and 
therapeutic implications. Cytokine Growth Factor Rev. 2006 17:441-450.

31. Oraby MA, El-Yamany MF, Safar MM, Assaf N, Ghoneim HA. Dapagliflozin attenuates early markers of diabetic nephropathy in fructose-streptozotocininduced diabetes in rats. Biomed Pharmacother. 2019;109:910-920.

32. Murty MSN, Sharma UK, Pandey VB, Kankare SB. Serum cystatin $\mathrm{C}$ as a marker of renal function in detection of early acute kidney injury. Indian $\mathrm{J}$. Nephrol. 2013;23:180-183.

33.Zhang S, Xu H, Yu X, Wang Y, Sun F, Sui D. Simvastatin ameliorates low-dose streptozotocininduced type 2 diabetic nephropathy in an experimental rat model. Int J Clin Exp Med. 2015 8:6388-6396.

34.Ni Z, Guo L, Liu F, Olatunji OJ, Yin M. Allium tuberosum alleviates diabetic nephropathy by supressing hyperglycemia-induced oxidative stress and inflammation in high fat diet/streptozotocin treated rats. Biomed Pharmacother. 2019;112:108678.

35.Zhang S, Xu H, Yu X, Wu Y, Sui D. Metformin ameliorates diabetic nephropathy in a rat model of low-dose streptozotocin-induced diabetes. Exp. Ther. Med. 2017;14:383-390.

36.Jaikumkao K, Pongchaidecha A, Chueakula N, Thongnak LO, Wanchai K, Chatsudthipong V et al. Dapagliflozin, a sodium-glucose co-transporter-2 inhibitor, slows the progression of renal complications through the suppression of renal inflammation, endoplasmic reticulum stress and apoptosis in prediabetic rats. Diabetes Obes Metab. 2018;20:26172626.
37.Pihl L, Persson P, Fasching A, Hansell P, DiBona GF, Palm F. Insulin induces the correlation between renal blood flow and glomerular filtration rate in diabetes: implications for mechanisms causing hyperfiltration. Am J Physiol Regul Integr Comp Physiol. 2012;303:R39-R47.

38. Fioretto P, Zambon A, Rossato M, Busetto L, Vettor R. SGLT2 Inhibitors and the Diabetic Kidney. Diabetes Care. 2016;2:S165-S171.

39. Patinha D, Fasching A, Pinho D, Albino-Teixeira An, Morato M, Palm F. Angiotensin II contributes to glomerular hyperfiltration in diabetic rats independently of adenosine type I receptors. Am J Physiol-Renal. 2013;304:F614-F622.

40. Navarro-González JF, Mora-Fernández C, de Fuentes MM, García-Pérez J. Inflammatory molecules and pathways in the pathogenesis of diabetic nephropathy. Nat. Rev. Nephrol. 2011;7:327-340.

41. Haywood JR, Shaffer RA, Fastenow C, Fink GD, Brody MJ. Regional blood flow measurement with pulsed Doppler flowmeter in conscious rat. Am J Physiol Heart Circ Physiol. 1981;241:H273-H278.

42. Freitas SCF, Harthmann ÂdA, Rodrigues B, Irigoyen M-C, De Angelis K. Effect of aerobic exercise training on regional blood flow and vascular resistance in diabetic rats. Diabetol Metab Syndr. $2015 ; 7: 115$.

43. Artunc F, Schleicher E, Weigert C, Fritsche A, Stefan N, Häring H-U. The impact of insulin resistance on the kidney and vasculature. Nat. Rev. Nephrol. 2016;12:721-737.

44. Asadi S, Goodarzi MT, Karimi J, Hashemnia M, Khodadadi I. Does curcumin or metformin attenuate 
oxidative stress and diabetic nephropathy in rats? J Nephropathol. 2019;8:8-8.

45. Qi H, Nielsen PM, Schroeder M, Bertelsen LB, Palm F, Laustsen C. Acute renal metabolic effect of metformin assessed with hyperpolarised MRI in rats. Diabetologia 2018 61:445-454.

46. Andrianesis V, Glykofridi S, Doupis J. The renal effects of SGLT2 inhibitors and a mini-review of the literature. Ther. Adv. Endocrinol. Metab. 2016;7:212228.

47. Freitas HS, Anhe GF, Melo KF, Okamoto MM, Oliveira-Souza $\mathrm{M}$, Bordin $\mathrm{S}$ et al. $\mathrm{Na}(+)$-glucose transporter-2 messenger ribonucleic acid expression in kidney of diabetic rats correlates with glycemic levels: involvement of hepatocyte nuclear factor-1alpha expression and activity. Endocrinology 2008;149:717724.
48.Jain D, Saha S. Antioxidant and antihyperglycaemic effects of naringenin arrest the progression of diabetic nephropathy in diabetic rats. Egypt. Pharm. J. 2017;16:144-151.

49. Rossing P, Frimodt-Moller M. Clinical Features and Natural Course of Diabetic Nephropathy. In: Roelofs JJ, Vogt L, editors. Diabetic Nephropathy: Pathophysiology and Clinical Aspects. Cham, Switzerland: Springer, 2019.

50.Chagnac A, Zingerman B, Rozen-Zvi B, HermanEdelstein M. Consequences of Glomerular Hyperfiltration: The Role of Physical Forces in the Pathogenesis of Chronic Kidney Disease in Diabetes and Obesity. Nephron 2019;143:38-42.

51.Tuttle KR. Back to the Future: Glomerular Hyperfiltration and the Diabetic Kidney. Diabetes. 2017;66:14-16.

To cite this article: Sohair A. Saleh , Magda A. Mansour , Suzan M. Hazzaa , Amal G. Younis, Dalia F. El Agamy. Dapagliflozin Ameliorates Glycemic State, Lipid Profile and Renal Functions in Type 2 Diabetic Rats. BMFJ 2020; 37(3): 636-652, DOI:10.21608/bmfj.2020.31557.1270 
Original article

DOI:10.21608/bmfj.2020.31557.1270 